\title{
Organ Volume Estimation Using SPECT
}

\author{
H. Zaidi
}

\begin{abstract}
Knowledge of in vivo thyroid volume has both diagnostic and therapeutic importance and could lead to a more precise quantification of absolute activity contained in the thyroid gland. In order to improve single-photon emission computed tomography (SPECT) quantitation, attenuation correction was performed according to Chang's algorithm. The dual window method was used for scatter subtraction. We used a Monte Carlo simulation of the SPECT system to accurately determine the scatter multiplier factor $k$. Volume estimation using SPECT was performed by summing up the volume elements (voxels) lying within the contour of the object, determined by a fixed threshold and the gray level histogram (GLH) method. Thyroid phantom and patient studies were performed and the influence of 1 ) fixed thresholding, 2) automatic thresholding, 3) attenuation, 4) scatter, and 5) reconstruction filter were investigated. This study shows that accurate volume estimation of the thyroid gland is feasible when accurate corrections are performed. The relative error is within $7 \%$ for the GLH method combined with attenuation and scatter corrections.
\end{abstract}

\section{INTRODUCTION}

$\mathbf{I}$ N DIAGNOSTIC and oncological nuclear medicine, singlephoton emission computed tomography (SPECT) is important for in vivo three-dimensional (3-D) imaging of radiopharmaceuticals. If the intention is to measure the volume and uptake in a specific organ, it may be convenient to make preliminary phantom studies on an object of similar shape and size. A phantom is used to simulate the geometry of the thyroid in the neck with the assumption that the size, shape, location, and density of the simulated thyroid and surrounding tissues are comparable to that of the patient to be measured. Absolute quantitation by SPECT is feasible and has been shown to be a clinically reliable and useful technique [1], [2]. The accurate determination of thyroid volume may be an important factor in the calculation of the most appropriate dose of therapeutic radioiodine [3]. The volume measurement of different organs using SPECT has been widely applied in the clinical field [4]-[8]. The major problems encountered in volume quantitation with SPECT are image segmentation and imperfect system transfer function. The difficulty in image segmentation is compounded by the low spatial resolution and high noise characteristics of SPECT images [9].

Long et al. [10] have evaluated the relative performance of a number of image segmentation methods for volume quantitation on simulated SPECT images. This included: 1) manual methods in which the operator defines the surface boundary of an image structure by delineating its crosssectional edges in the contiguous slices incorporating it, 2)

Manuscript received September 27, 1995; revised January 24, 1996.

The author was with the Department of Medical Physics, Centre de Radioprotection et de Sûreté, Alger, Algeria. He is now with the Division of Nuclear Medicine, Geneva University Hospital, CH-1211, Geneva, Switzerland.

Publisher Item Identifier S 0018-9499(96)04844-7. algorithms based on simple thresholding techniques to define the edge of the organ where voxels are flagged as part of the volume of interest if they contain counts greater than some pre-defined threshold value, 3) the gray level histogram (GLH) method, which uses a nonparametric procedure of automatic threshold selection formulated by Otsu [11], and 4) twodimensional (2-D) and 3-D implementations of a multifeature determination of edge location based on calculation of the gradient (magnitude and direction of the greatest rate of change in counts). They conclude that the 3-D gradientbased method of image segmentation is the most useful. However, this method is time-consuming if implemented on a general purpose nuclear medicine computer system for use in clinical routine. The aim of this paper is to compare the relative performance of the fixed-threshold method and the GLH method and to study the influence of different correction techniques (attenuation, scatter) on thyroid volume quantitation.

\section{MATERIALS AND METHODS}

\section{A. Data Acquisition and Processing}

Imaging was performed using ${ }^{99 m} \mathrm{Tc}$ pertechnetate and a commercial scintillation camera system (Toshiba GCA $901 \mathrm{~A} / \mathrm{ECT}$ ), equipped with a low-energy general purpose parallel-hole collimator (hexagonal holes, hole diameter $=$ $2.36 \mathrm{~mm}$, septum wall thickness $=0.22 \mathrm{~mm}$, and collimator thickness $=40 \mathrm{~mm}$. The NaI(Tl) crystal measured $500 \times$ $400 \times 9.53 \mathrm{~mm}$. The energy resolution of the detector was $12.6 \%$ full width at half maximum (FWHM) for $140 \mathrm{keV}$ and the intrinsic spatial resolution was about $3.8 \mathrm{~mm}$. Sixty frames were acquired in step-and-shoot mode over $360^{\circ}$ with an acquisition time of $30 \mathrm{~s}$ per angle.

Projection data were acquired using two pulse-height windows (127-153 keV and $92-125 \mathrm{keV})$ to include the photopeak and Compton scatter, respectively, and were corrected for radioactive decay. About 100 kcounts per projection, on average, were obtained for the various phantoms. After nine-point smoothing, transaxial images of one pixel thickness were reconstructed into a $128 \times 128$ matrix using a filtered backprojection algorithm. A third-order Butterworth filter having a cut-off frequency equal to 0.4 Nyquist was used. Standard quality control procedures were performed regularly using a protocol suggested by the National Electrical Manufacturers Association [12], which includes the determination of pixel size, acquisition of flood fields for uniformity correction, and the determination of the center of rotation. The radius of rotation used for phantom studies is $14 \mathrm{~cm}$. The spatial resolution was measured as $12.1 \mathrm{~mm}$ FWHM using our 
acquisition and processing protocol. Measurement of pixel size yielded a value of $0.4 \mathrm{~cm}$.

\section{B. Attenuation and Scatter Corrections}

The quantitative information in SPECT images is distorted by photon attenuation and contribution of photons scattered in the object. In order to improve the accuracy of SPECT quantitation, correction methods have to be used. A simple approach to compensating for scatter is to use a smaller value for the linear attenuation coefficient, such as $0.12 \mathrm{~cm}^{-1}$. By this approach, one compensates for scatter by undercorrecting for attenuation as it occurs in the medium. In the second approach, the Compton scattering elimination is carried out by a modified version of the dual window method [13]. In this way, the scattering was corrected on the projections instead of on the slices. Scatter subtraction substantially improves the final impulse response and the resolution of the slices. A postprocessing attenuation correction algorithm [14] assuming a uniform attenuation with a linear attenuation coefficient of $0.15 \mathrm{~cm}^{-1}$ was applied in case of combination of attenuation and scatter corrections and with an effective linear attenuation coefficient of $0.12 \mathrm{~cm}^{-1}$ when the attenuation correction is applied alone. It was also decided to reconstruct the images without any correction since this would give an indication of the worst situation. We used a Monte Carlo simulation of the SPECT system [15] to accurately determine the scatter multiplier factor $k$ required for scatter correction. Unlike the experimental devices, the computer simulation is able to distinguish independently between scattered and nonscattered events. Thus, the true number of scattered events recorded within the photopeak may be determined. The dimensions and characteristics of the SPECT system together with the phantom used for the measurements and the data acquisition parameters were used as input for the computer code (SIMIND), which resulted in a value of 0.42 for the weighting factor $k$ for our gamma camera system and reconstruction algorithm. This value is smaller than the one found in [16], which is probably due to the smaller size of the cylinder used in the experiments and also the difference between the characteristics of the two SPECT systems. The results presented in the next section were obtained using this computer-simulated value.

\section{Phantom Studies}

Volume quantitation using SPECT was performed by summing up the volume elements (voxels) lying within the contour of the phantom determined by fixed thresholding (FT) and adaptive thresholding (GLH method) in each reconstructed slice. A detailed description of the algorithm of GLH method used has been given by [11] and [17]. Briefly, the method entailed the following steps. The operator has to define a region of interest ( $\mathrm{ROI}$ ) so that only the selected object is included. A gray-level histogram of the pixels in the selected $\mathrm{ROI}$ in all the slices is constructed. The method of maximizing the interclass variance is then applied to obtain the threshold value that optimally separated pixels within and without the object. It has been shown that the size of the ROI only slightly influences the final result [17].
Fifteen thyroid phantoms with volumes ranging from 16 to $75 \mathrm{ml}$ were studied: 14 combinations of ellipsoids and a 3$\mathrm{D}$ realistic thyroid phantom (MIRD mathematical phantom). For greater realism, these phantoms were placed in a solid, homogeneous perspex cylindrical phantom (diameter $=90$ $\mathrm{mm}$, height $=240 \mathrm{~mm}$ ) filled with nonradioactive water at a depth approximately equal to the average gland depth in the patient's neck. The effects of geometry, attenuation, and scatter are presumed to be the same for the phantom and the patient. Each phantom was filled with water to which was added ${ }^{99 m} \mathrm{TcO}_{4}^{-}$to give an activity concentration of $74 \mathrm{kBq} / \mathrm{ml}(2 \mu \mathrm{Ci} / \mathrm{ml})$. This value was chosen to mimic the activity concentration generally encountered in clinical practice. The volume (y) was then calculated by the two methods (FT and GLH) with and without the correction methods described above, and correlation with the actual volume $(x)$ was checked.

\section{Clinical Studies}

Subsequently, seven patients were studied who were referred to the Nuclear Medicine Department of Malmö General Hospital for thyroid evaluation. Two hundred MBq of ${ }^{99 m} \mathrm{Tc}$ pertechnetate were administered intravenously to each patient, and acquisition started 20-30 min later. In this case, an elliptical orbit was used instead of a circular one. Reconstruction was done in a manner identical to that used for the phantom studies. Additional sets of images were generated by reconstructing the patient studies using a Shepp and Logan filter and a fourth-order Butterworth filter having a cut-off frequency of 0.25 Nyquist in order to assess the influence of the reconstruction filter on volume quantitation. Attenuation correction was performed according to Chang's algorithm with an effective linear attenuation coefficient of $0.12 \mathrm{~cm}^{-1}$.

\section{RESULTS}

Fig. 1(a) illustrates the results of classical filtered backprojection computed with the photopeak data for a thyroid phantom. The first-order attenuation correction with $\mu=$ $0.12 \mathrm{~cm}^{-1}$ appears in Fig. 1(b). The benefits of simultaneous Compton and attenuation compensation $\left(\mu=0.15 \mathrm{~cm}^{-1}\right)$ is clearly shown in Fig. 1(c). Figs. 2-5 show the correlation between the true and the calculated volumes with and without the different correction techniques for fixed threshold values of $30 \%, 35 \%$, and $40 \%$ and for the GLH method, respectively. The line connecting the data points represents the results of a linear regression analysis. From our analysis, we found that the threshold required for accurate edge location is a function of both the source size and whether or not attenuation and scatter correction methods are performed [18]. For example, when the source volume is $26 \mathrm{ml}$, the threshold required for accurate edge location is $42 \%$ without any correction, $40 \%$ with attenuation correction, and $37.5 \%$ with attenuation and scatter corrections. Count profiles through each slice showed that because of the uniform distribution of the radioactivity in the phantoms, the variation in the maximum pixel value in each slice was small and close to the average intensity value of the central area of each appropriate slice. 


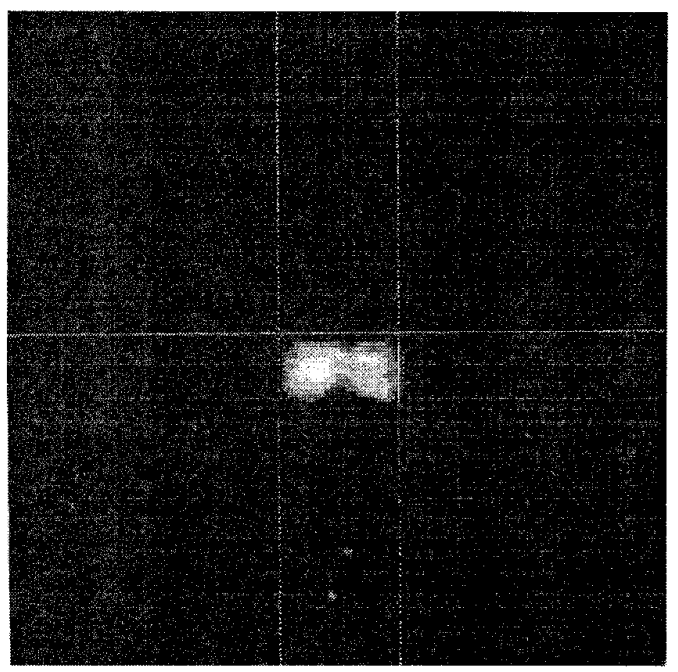

(a)

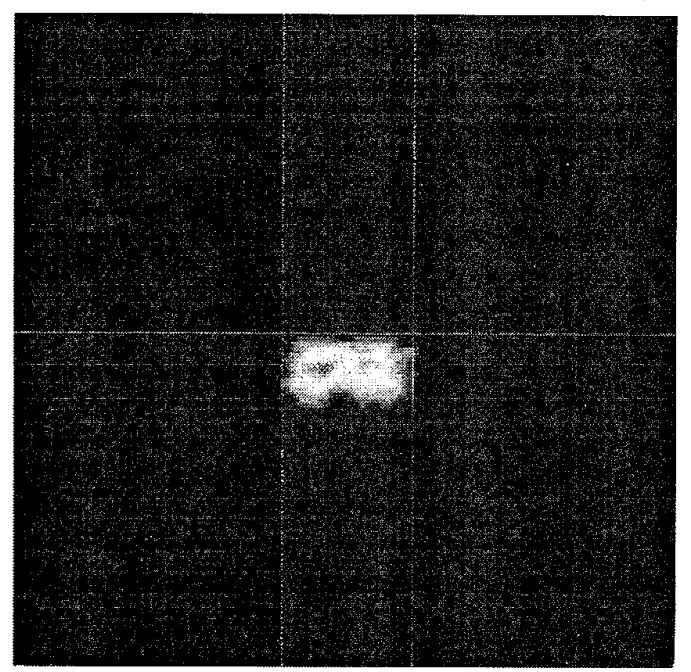

(b)

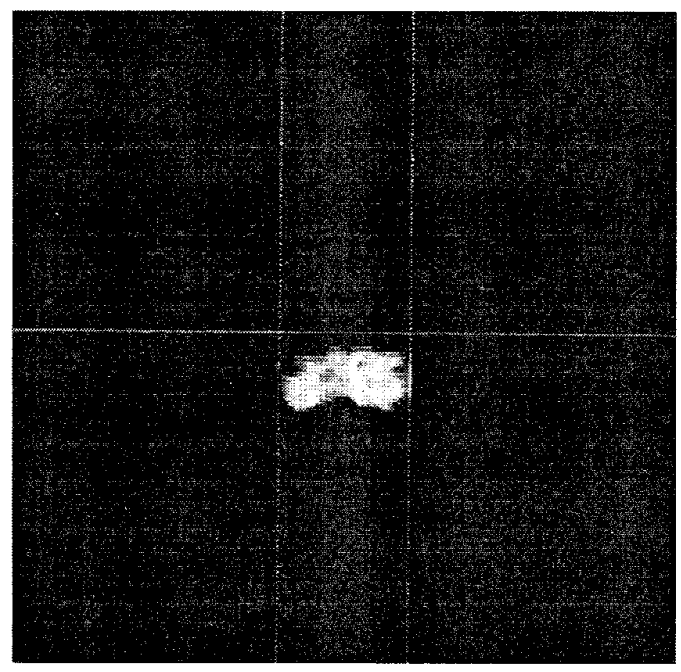

(c)

Fig. 1. Example of the effect of attenuation and scatter compensation. (a) Reconstructed image of a thyroid phantom obtained from filtered backprojection without correction. (b) Same image corrected for attenuation using Chang's method $\left(\mu=0.12 \mathrm{~cm}^{-1}\right)$. (c) Reconstructed image after performing attenuation and scatter corrections

TABLE I

COMPARISON OF THE RELATIVE ERRORS (\%) BETWEEN THE TRUE aNd THE CALCULATED VOLUMES, WHICH ARE DEFINED AS THE Absolute Values of the Difference BetweEn the TRue and the Calculated Volumes Divided by the True Volume. Mean Relative Errors with Standard Deviations are Shown

\begin{tabular}{lcccc}
\hline & Th=30\% & Th=35\% & Th=40\% & GLH method \\
\hline $\mathrm{NC}^{1}$ & $35.2 \pm 34.8$ & $17.9 \pm 26.1$ & $20.2 \pm 17.2$ & $20.8 \pm 6.8$ \\
$\mathrm{CA}^{2}$ & $29.7 \pm 34.5$ & $17.4 \pm 23.6$ & $22.3 \pm 15.8$ & $16.4 \pm 5.8$ \\
$\mathrm{CAS}^{3}$ & $18.6 \pm 25.6$ & $16.8 \pm 17.9$ & $29.1 \pm 9.4$ & $7.3 \pm 4$ \\
\hline
\end{tabular}

not corrected

corrected for attenuation

3 corrected for attenuation and scater

A comparison of the relative errors between the true and calculated volumes for the different methods are summa- rized in Table I. Table II illustrates a comparison of thyroid volume assessments for seven patients with and without attenuation correction and with different reconstruction filters. No important variation was observed when using different reconstruction filters, however the comparison of the values obtained from attenuation corrected and noncorrected images resulted in a mean difference of $4.6 \%, 3.8 \%$, and $3.4 \%$ using a fixed threshold value of $30 \%, 40 \%$, and the GLH method, respectively, when a third-order Butterworth filter is used.

\section{Discussion}

Thyroid volume can be a significant clue to the presence of disease, and changes in its size can be important indicators of the therapeutic effect. Rotating gamma-camera SPECT systems are ideal for such volumetric quantification because they are true 3-D volume-imaging systems. Filtered back- 


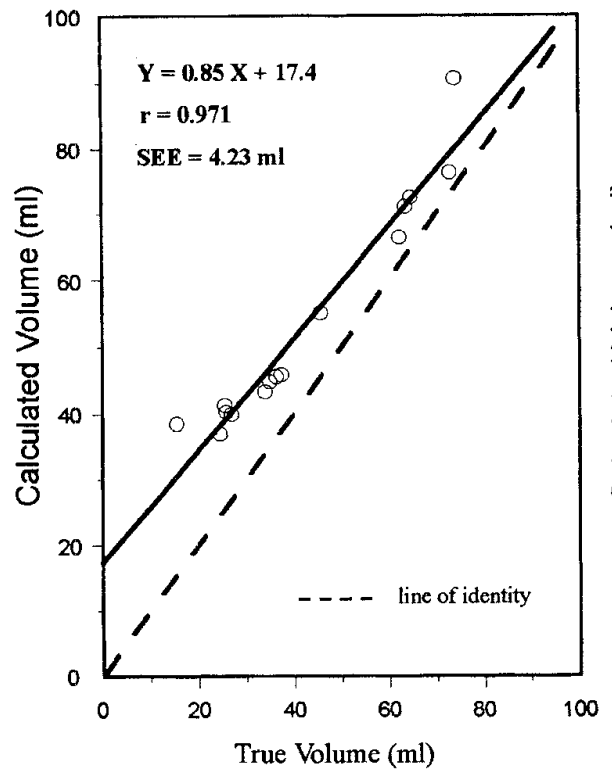

(a)

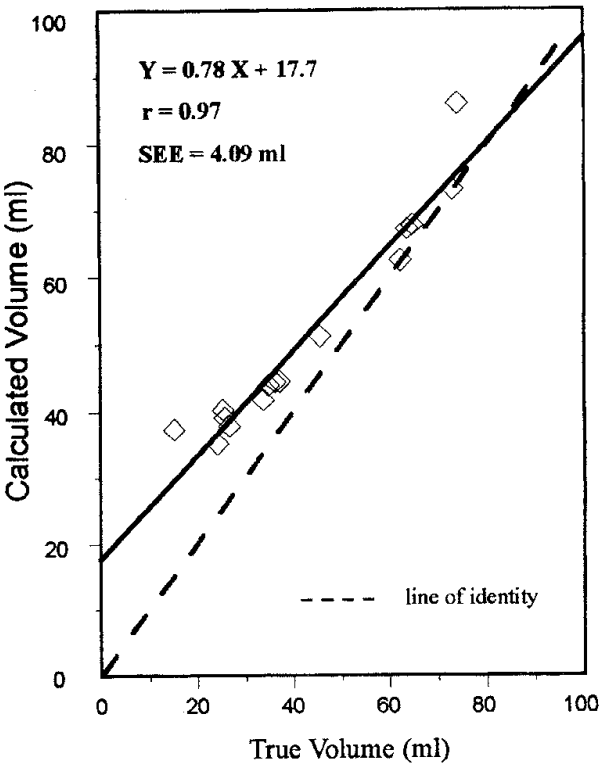

(b)
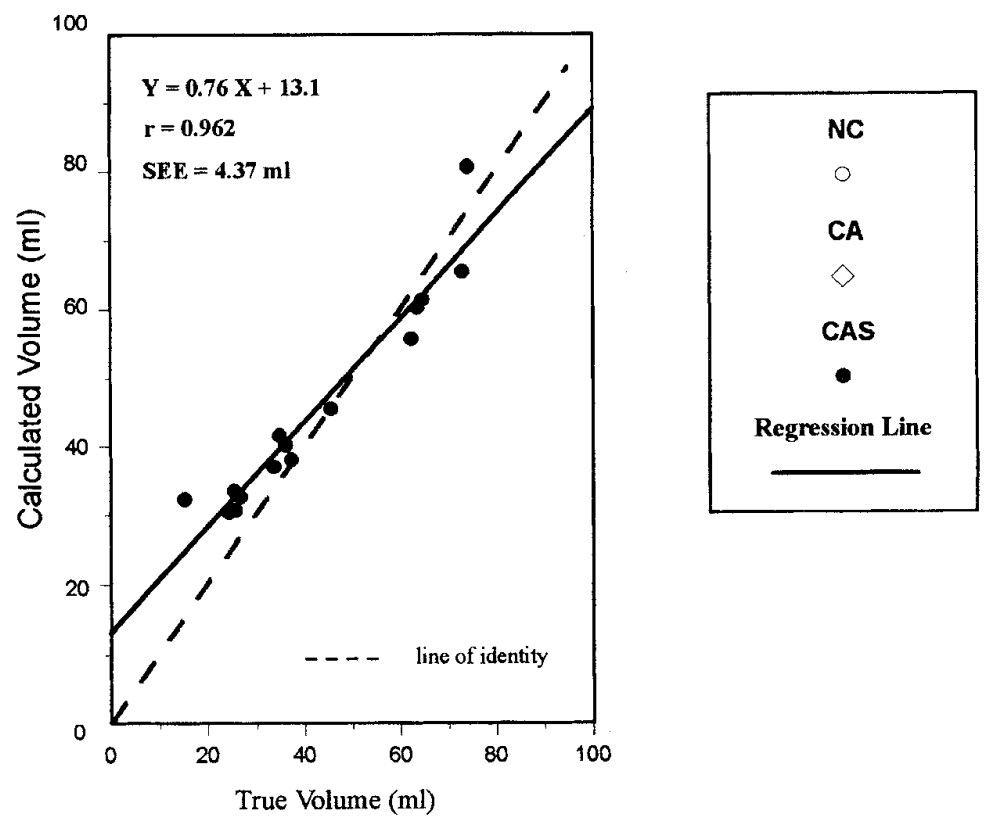

(c)

Fig. 2. Correlation between the true and the calculated volumes for a fixed threshold value of $30 \%$ (a) without attenuation correction (NC), (b) with attenuation correction (CA), and (c) with attenuation and scatter corrections (CAS).

projection after dual window scatter subtraction followed by Chang attenuation correction leads to satisfactory quantitative accuracy (even with first-order attenuation compensation).

A number of factors influence the accuracy of volume estimation with SPECT imaging when one uses threshold edge detection methods. As stated by many authors, no single threshold is ideal for all object shapes and sizes, and each group picked the threshold that best matched their specific phantom studies and imaging system [1], [6]. Fig. 2 illustrates the experimental results obtained using a fixed threshold value of $30 \%$. It has been shown that there is an overestimation of the true volume for all phantoms with a fixed threshold of $30 \%$ without or with attenuation correction. For a threshold of $35 \%$ or $40 \%$ (Figs. 3 and 4 ), we found an overestimation of the smallest volumes and an underestimation of the largest volumes. As the source size increases, the threshold required to yield accurate edge detection decreases. Thus, these methods were inaccurate. It was demonstrated in the study of [9] that the threshold required for accurate volume quantitation depends on object shape and size relative to the system spatial resolution.

The adaptive threshold or GLH method [17], [19] attempts to circumvent the problem of applying a single fixed threshold 


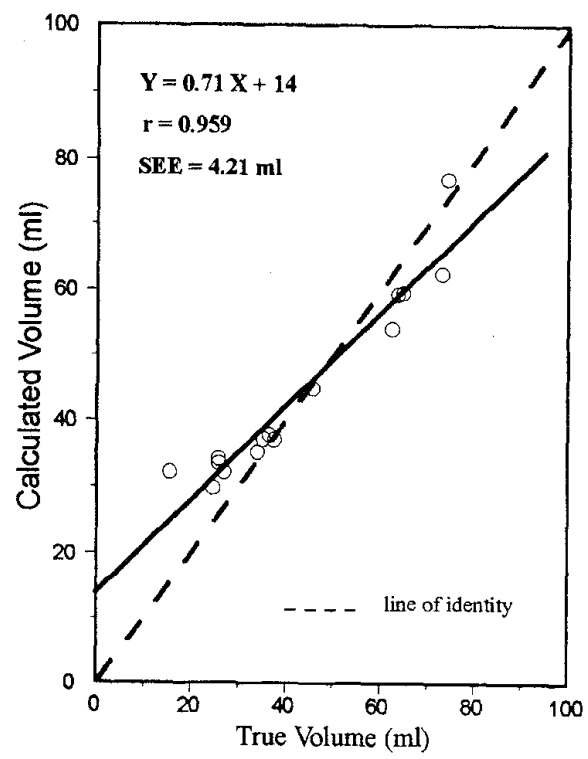

(a)

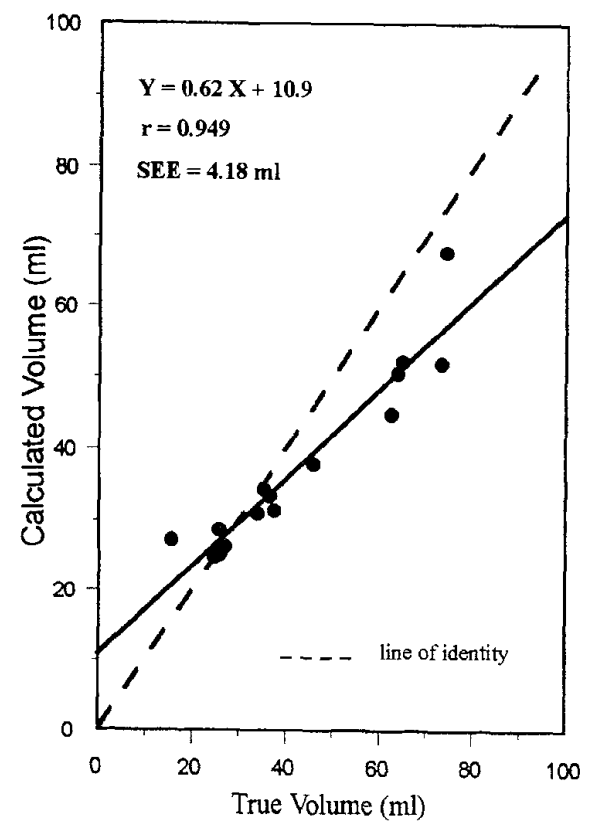

(c)

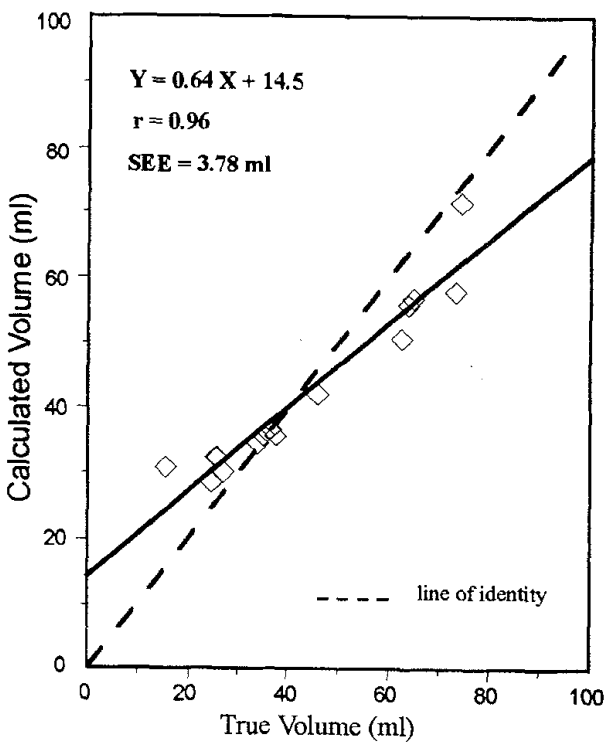

(b)

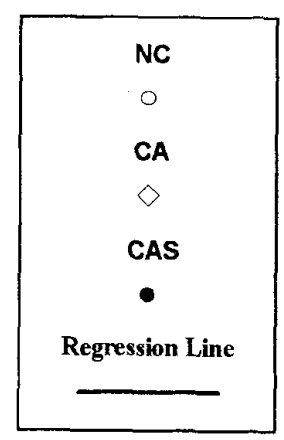

Fig. 3. Correlation between the true and the calculated volumes for a fixed threshold value of $35 \%$ (a) without attenuation correction (NC), (b) with attenuation correction (CA), and (c) with attenuation and scatter corrections (CAS).

by adapting the threshold to each object based on the graylevel histogram in the region surrounding the object. This method, however, is sensitive to both noise and contrast and produced appreciable errors in volume estimation unless simultaneous correction for attenuation and scatter are included in the method (Table I). In our case, there was an excellent correlation $(r=0.99)$ between the true $(x)$ and the calculated volume $(y)$, the regression line agreed well with the line of identity (Fig. 5), and the volume of the thyroid phantoms is estimated with approximately $7 \%$ accuracy when using the
GLH method combined with attenuation and scatter correction techniques, which is quite satisfactory compared to the results obtained with the other methods (Table I). The increase in accuracy may be due to the high contrast reached after Compton and attenuation compensation, which allows better distinction between the thyroid phantoms and the surrounding medium in the GLH, so that the statistical method could determine an optimal threshold. The GLH method combined with attenuation and scatter corrections was shown to provide quantitation and contrast results that were superior to the 


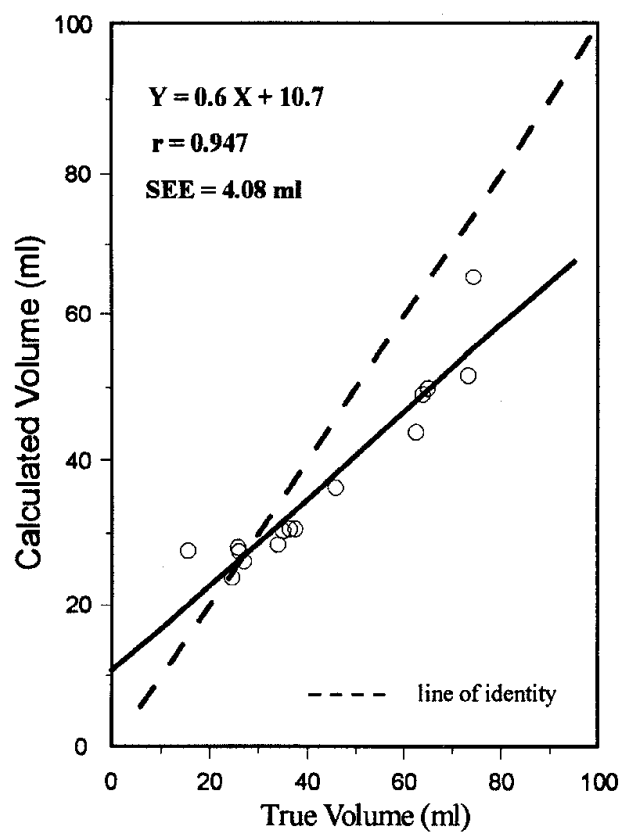

(a)

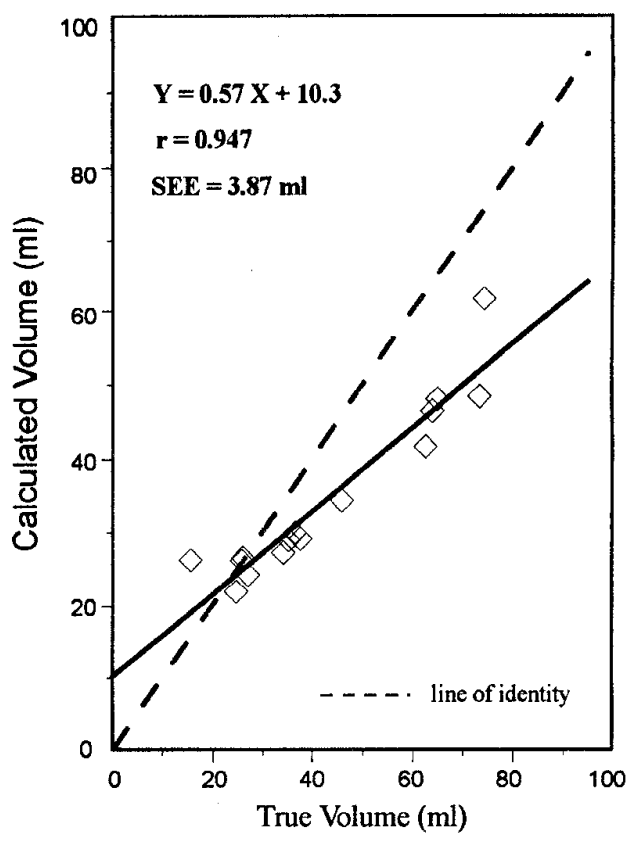

(b)
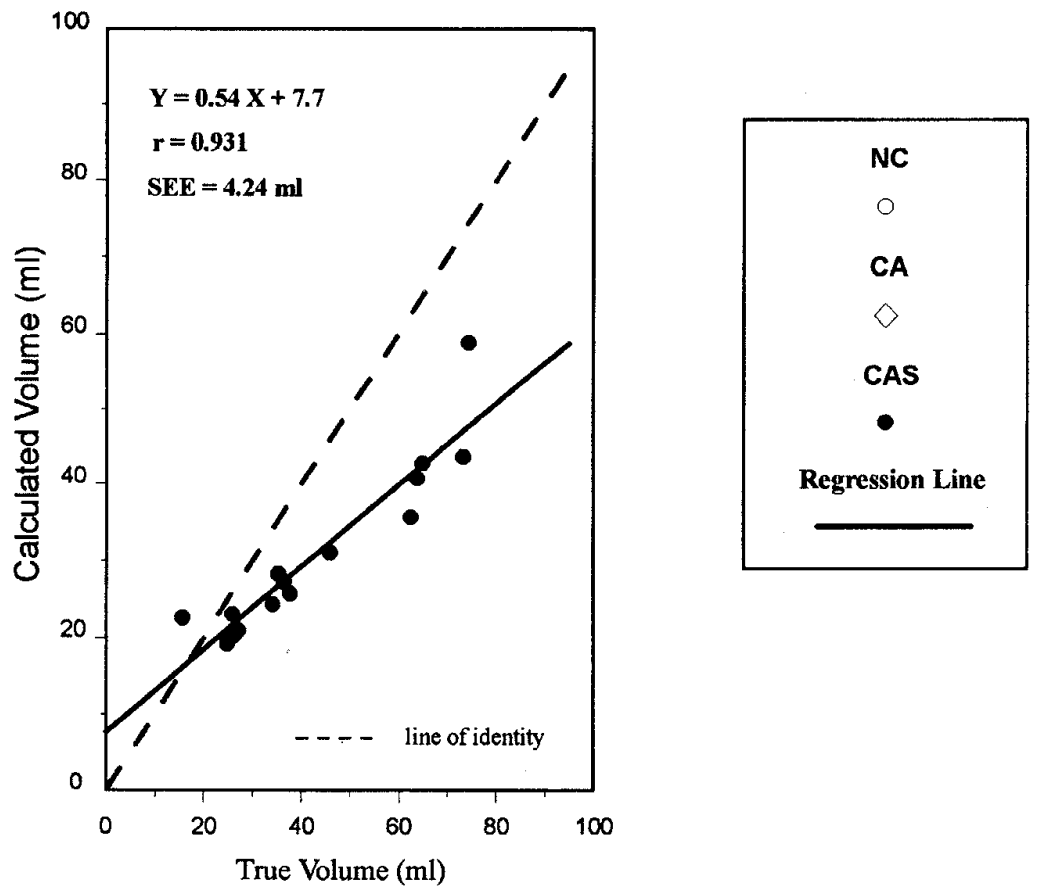

(c)

Fig. 4. Correlation between the true and the calculated volumes for a fixed threshold value of $40 \%$ (a) without attenuation correction (NC), (b) with attenuation correction (CA), and (c) with attenuation and scatter corrections (CAS).

simple method of scatter correction using a reduced value of $\mu$ in the attenuation correction. This should, however, be regarded as a second-best approach to the problem.

The reconstruction filter is often chosen on a semi-objective basis. However, subjective evaluation of studies from patients known to have thyroid disease based upon other diagnostic procedures demonstrated that the 0.4 Nyquist cut-off value of the third-order Butterworth filter produced the best image quality. The volume calculation showed no significant difference when using different reconstruction filters (Table II).

The developed method works fairly well with the phantom volumes used $(16-75 \mathrm{ml})$. An overestimation of smaller volumes is expected due to the limitations of the spatial resolution of the SPECT system. So, meaningful and con- 


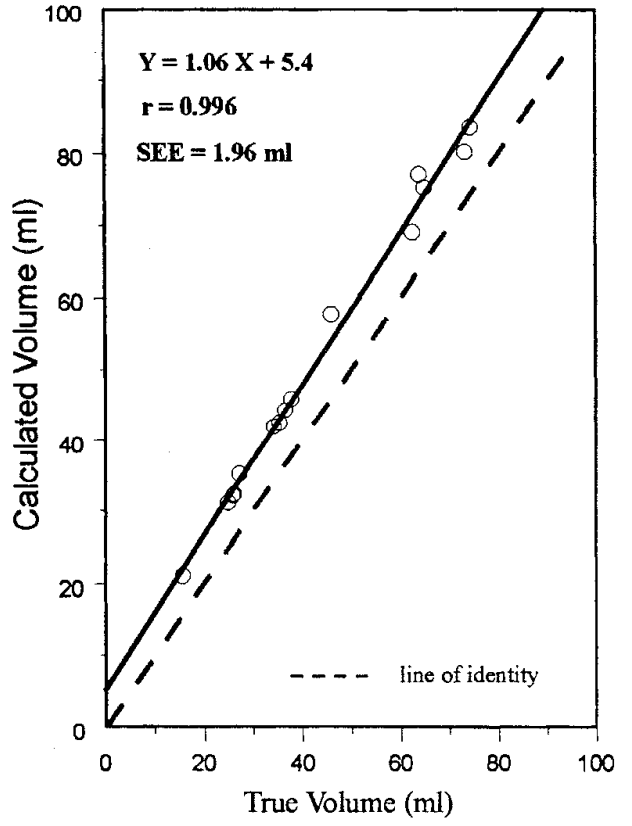

(a)

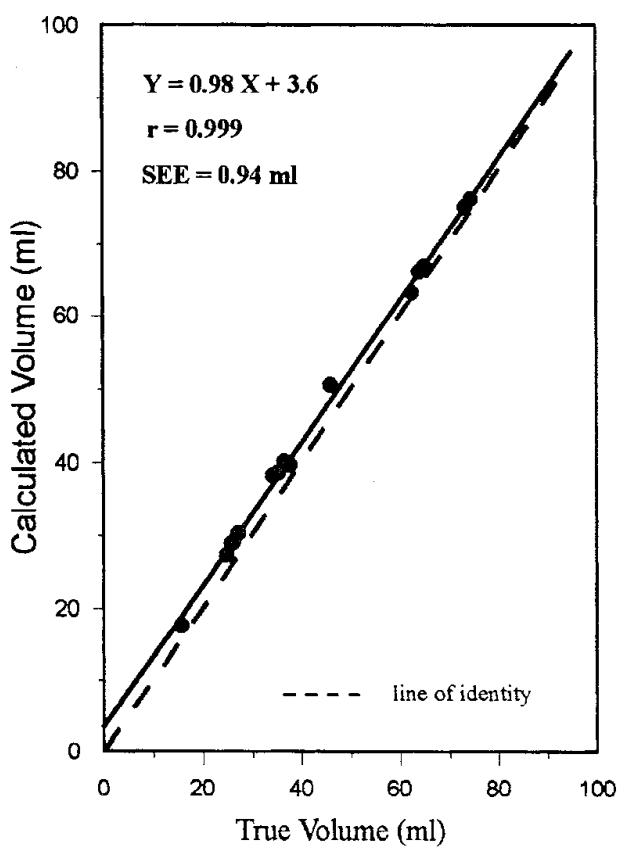

(c)

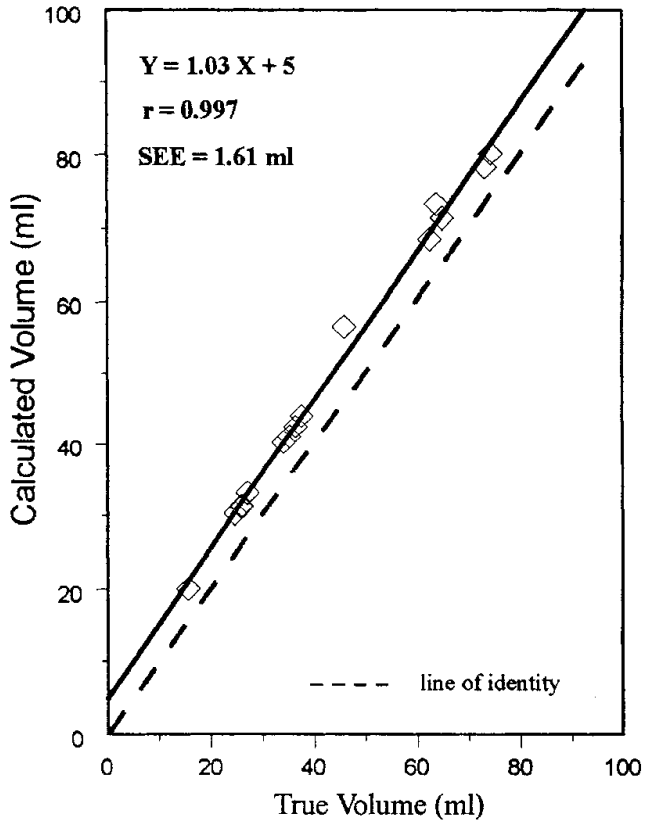

(b)

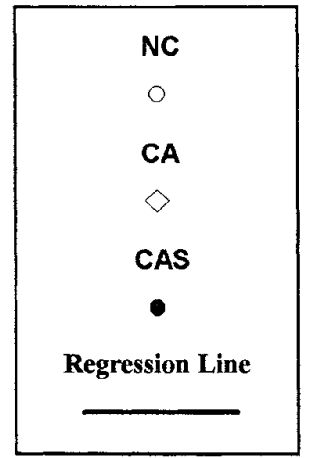

Fig. 5. Correlation between the true and the calculated volumes for the GLH method (a) without attenuation correction (NC), (b) with attenuation correction (CA), and (c) with attenuation and scatter corrections (CAS).

sistent results could not be obtained due to the small size of the object relative to the system's spatial resolution. If our goal is to improve the spatial resolution, the geometric resolution of the collimator is of concern. In SPECT, the choice of collimator involves a trade-off between sensitivity and spatial resolution. Improvement in resolution leads to losses in sensitivity proportional to the square of the FWHM. Webb et al. [20] used SPECT and a fan-beam collimator for measuring thyroid volume with the explicit aim of improving spatial resolution without sacrificing sensitivity. The volume sensitivity was some $30 \%$ greater than for a corresponding low-energy general purpose parallel-hole collimator. For their segmentation technique, volume errors of approximately $15 \%$ were reported. Scintillation cameras fitted with pinhole collimators are particularly suited for tomographic imaging of small objects such as the thyroid gland since these collimators 
TABLE II

Measured Thyroid Vol.jme with and Without Attenuation Correction and with Different Reconstruction Filters

\begin{tabular}{|c|c|c|c|c|c|c|c|c|c|}
\hline \multirow[b]{2}{*}{$\begin{array}{c}\text { Patient } \\
\text { no (sex, age) }\end{array}$} & \multicolumn{3}{|c|}{$\mathrm{Th}=30 \%$} & \multicolumn{3}{|c|}{ Th $=40 \%$} & \multicolumn{3}{|c|}{ GLH method } \\
\hline & $\begin{array}{l}\mathrm{NC} \\
(\mathrm{ml}) \\
\end{array}$ & $\begin{array}{l}\mathrm{CA} \\
(\mathrm{ml}) \\
\end{array}$ & $\%$ Diff & $\begin{array}{l}\text { NC } \\
(\mathrm{ml}) \\
\end{array}$ & $\begin{array}{l}\mathrm{CA} \\
(\mathrm{ml}) \\
\end{array}$ & $\%$ Diff & $\begin{array}{l}\mathrm{NC} \\
(\mathrm{ml})\end{array}$ & $\begin{array}{l}\mathrm{CA} \\
(\mathrm{ml})\end{array}$ & $\%$ Diff \\
\hline $\begin{array}{l}\text { 1F, } 75 \\
\text { S-L } \\
\text { BW4 } 0.25 W^{5} \\
\text { BW3 } 0.4 W^{6}\end{array}$ & $\begin{array}{l}50.2 \\
50.8 \\
50.3 \\
\end{array}$ & $\begin{array}{l}50.7 \\
47.2 \\
46.9 \\
\end{array}$ & $\begin{array}{l}0.1 \\
7.1 \\
6.8 \\
\end{array}$ & $\begin{array}{c}30.44 \\
30.5 \\
30.5 \\
\end{array}$ & $\begin{array}{l}27.6 \\
28.7 \\
28.2 \\
\end{array}$ & $\begin{array}{l}9.1 \\
5.9 \\
7.5 \\
\end{array}$ & $\begin{array}{l}43.7 \\
43.4 \\
42.9 \\
\end{array}$ & $\begin{array}{l}40.7 \\
42.1 \\
41.5 \\
\end{array}$ & $\begin{array}{l}6.8 \\
2.9 \\
3.3 \\
\end{array}$ \\
\hline $\begin{array}{l}2 F, 46 \\
\text { S-L } \\
\text { BW4 } 0.25 W \\
\text { BW3 } 0.4 W \\
\end{array}$ & $\begin{array}{c}49.8 \\
50.9 \\
50 \\
\end{array}$ & $\begin{array}{l}46.8 \\
47.4 \\
47.3 \\
\end{array}$ & $\begin{array}{c}6 \\
6.9 \\
5.5 \\
\end{array}$ & $\begin{array}{c}29 \\
30.4 \\
29 \\
\end{array}$ & $\begin{array}{l}29.2 \\
28.1 \\
27.1 \\
\end{array}$ & $\begin{array}{l}0.8 \\
7.5 \\
6.7 \\
\end{array}$ & $\begin{array}{l}32.4 \\
32.7 \\
31.6 \\
\end{array}$ & $\begin{array}{l}30.3 \\
30.3 \\
29.9 \\
\end{array}$ & $\begin{array}{l}5.7 \\
6.5 \\
5.4 \\
\end{array}$ \\
\hline $\begin{array}{l}3 F, 69 \\
\text { S-L } \\
\text { BW4 } 0.25 W \\
\text { BW3 } 0.4 W \\
\end{array}$ & $\begin{array}{c}45.9 \\
46.4 \\
46 \\
\end{array}$ & $\begin{array}{l}43.6 \\
44.2 \\
44.2 \\
\end{array}$ & $\begin{array}{c}5 \\
4.8 \\
3.9 \\
\end{array}$ & $\begin{array}{c}29 \\
29.2 \\
29.2 \\
\end{array}$ & $\begin{array}{l}28.1 \\
27.8 \\
28.3 \\
\end{array}$ & $\begin{array}{c}3.2 \\
5 \\
3.3 \\
\end{array}$ & $\begin{array}{r}33.5 \\
35.2 \\
35 \\
\end{array}$ & $\begin{array}{l}34.7 \\
33.8 \\
34.6 \\
\end{array}$ & $\begin{array}{l}3.6 \\
3.9 \\
0.9 \\
\end{array}$ \\
\hline $\begin{array}{l}\text { 4F, } 59 \\
\text { S-L } \\
\text { BW4 } 0.25 W \\
\text { BW3 } 0.4 W \\
\end{array}$ & $\begin{array}{c}34 \\
34.6 \\
34.1 \\
\end{array}$ & $\begin{array}{l}32.1 \\
32.3 \\
31.7 \\
\end{array}$ & $\begin{array}{l}5.6 \\
6.5 \\
6.8 \\
\end{array}$ & $\begin{array}{c}22 \\
22.4 \\
22.1 \\
\end{array}$ & $\begin{array}{c}21.4 \\
22 \\
21.1 \\
\end{array}$ & $\begin{array}{l}2.8 \\
1.6 \\
4.3 \\
\end{array}$ & $\begin{array}{l}28.1 \\
27.9 \\
27.6 \\
\end{array}$ & $\begin{array}{c}28.3 \\
27.1 \\
28 \\
\end{array}$ & $\begin{array}{l}0.7 \\
2.7 \\
1.5 \\
\end{array}$ \\
\hline $\begin{array}{l}\mathbf{5 F}, \mathbf{3 8} \\
\text { S-L } \\
\text { BW4 } 0.25 W \\
\text { BW3 } 0.4 W\end{array}$ & $\begin{array}{l}47.7 \\
48.3 \\
47.8 \\
\end{array}$ & $\begin{array}{l}46.5 \\
47.1 \\
47.1\end{array}$ & $\begin{array}{l}2.4 \\
2.5 \\
1.5\end{array}$ & $\begin{array}{l}31.5 \\
32.1 \\
31.7\end{array}$ & $\begin{array}{l}30.9 \\
31.7 \\
31.3\end{array}$ & $\begin{array}{l}2.1 \\
1.1 \\
1.1\end{array}$ & $\begin{array}{r}34.9 \\
34.8 \\
32.2 \\
\end{array}$ & $\begin{array}{l}32.3 \\
32.1 \\
30.5 \\
\end{array}$ & $\begin{array}{l}7.5 \\
7.8 \\
5.4 \\
\end{array}$ \\
\hline $\begin{array}{l}\text { 6F, } 39 \\
\text { S-L } \\
\text { BW4 } 0.25 W \\
\text { BW3 } 0.4 W \\
\end{array}$ & $\begin{array}{l}19.2 \\
19.4 \\
19.2 \\
\end{array}$ & $\begin{array}{l}18.9 \\
19.1 \\
18.7 \\
\end{array}$ & $\begin{array}{l}1.3 \\
1.8 \\
2.3 \\
\end{array}$ & $\begin{array}{l}12.4 \\
12.7 \\
12.5 \\
\end{array}$ & $\begin{array}{l}11.9 \\
12.5 \\
12.3 \\
\end{array}$ & $\begin{array}{c}4 \\
1.1 \\
2 \\
\end{array}$ & $\begin{array}{l}18.9 \\
18.5 \\
18.4 \\
\end{array}$ & $\begin{array}{l}18.3 \\
17.8 \\
18.7 \\
\end{array}$ & $\begin{array}{c}3.3 \\
3.7 \\
2 \\
\end{array}$ \\
\hline $\begin{array}{l}7 \mathbf{F}, 42 \\
\text { S-L } \\
\text { BW4 } 0.25 W \\
\text { BW3 } 0.4 W \\
\end{array}$ & $\begin{array}{l}44.8 \\
44.8 \\
44.3 \\
\end{array}$ & $\begin{array}{l}42.4 \\
42.5 \\
41.9 \\
\end{array}$ & $\begin{array}{l}5.3 \\
5.1 \\
5.4\end{array}$ & $\begin{array}{c}26.2 \\
26.9 \\
26 \\
\end{array}$ & $\begin{array}{l}25.9 \\
26.2 \\
25.6 \\
\end{array}$ & $\begin{array}{l}1.3 \\
2.6 \\
1.4 \\
\end{array}$ & $\begin{array}{l}32.1 \\
31.3 \\
32.2 \\
\end{array}$ & $\begin{array}{l}30.2 \\
29.8 \\
30.4 \\
\end{array}$ & $\begin{array}{l}5.9 \\
4.8 \\
5.6 \\
\end{array}$ \\
\hline
\end{tabular}

${ }^{4}$ Shepp and Logan filter

${ }^{5}$ Fourth order Butterworth filter with cut-off frequency of 0.25 Nyquist

${ }^{6}$ Third order Butterworth filter with cut-off frequency of 0.4 Nyquist

combine high spatial resolution with magnification and small variation of resolution with distance [21]. However, problems exist, such as variations in the magnification and sensitivity with object to collimator distance and image distortions due to the finite size of the pinhole diameter. The major drawback of pinhole SPECT is that a special reconstruction program has to be used, which is not generally available in nuclear medicine departments.

The use of PET in the measurement of thyroid volume has already been reported in [3]. The error on the volume estimate was reduced to $\sim 4 \%$ for all volumes in the range $20-70 \mathrm{ml}$ and to $\sim 10 \%$ for volumes down to $2 \mathrm{ml}$. However, the high cost of PET and the need for an on-site cyclotron and radiochemical/radiopharmaceutical support has limited PET to very few clinical sites. In most of the investigations reported in the literature, the accuracy of volume quantitation using SPECT has been validated by phantom studies. It is evident that many differences appear between determining the size of phantoms in physical experiments and determining the size of human organs in vivo. The use of simulating phantoms is fraught with error because it is impossible to obtain an accurate simulation of the distribution of activity for each patient, even with the most elaborate phantom. However, this evaluation is useful for thyroid studies only because the gland depth and size do not differ by much among most subjects.
The accuracy reached in phantom studies in unlikely to be reached in clinical investigations. Animal studies and surgery samples taken from patients treated with total ablation of the thyroid gland after SPECT imaging was performed is a more straightforward approach for validation of phantom data. Since patients' necks vary in size, it would be useful to repeat the measurements with different-size cylinders.

Although reasonably accurate volume measurements can, in fact, be performed using SPECT, higher resolution imaging modalities such as computed tomography (CT), magnetic resonance imaging (MRI) [22], or ultrasound (US) [23] may be preferable for measuring the volume for many tumours, whose linear dimensions often approach the system spatial resolution. However, CT-, MRI-, and US-derived volumes need not be necessarily the same as the volumes in which the radionuclide localize (localization volumes), because the physiological uptake may not correspond exactly to the anatomical configuration of an organ. The difference in the second point arises from the fact that it is the functional and not the physical volume that is measured with SPECT. Since the functioning volume of the thyroid will generally be different from the anatomical volume measured, this can only be determined by radionuclide imaging techniques.

In this study, all the phantom acquisitions were performed without the presence of background activity. We expect a decrease in accuracy as the contrast decreases [10], [24]. However, according to the patients studied, the biological background as displayed on the SPECT images was not significant. Although the method could be adapted for routine use, it may, however, not be applicable to clinical situations that contain poor contrast and a high background activity [24]. The dependence of systematic error on other parameters such as contrast and noise is not yet evaluated.

Because of the need for evaluation in a clinical environment, we applied these volume measurements to calculate the radioactive dose required for hyperthyroid patients. The main objective in dose planning is the determination of the absorbed dose in the target per unit of activity administered, Gy/MBq. With quantitative SPECT, this ratio can be accurately calculated, and it may be possible to determine the activity of the "therapeutic" radiopharmaceutical necessary to give the required absorbed dose to the target [2].

\section{Conclusion}

The GLH method combined with attenuation and scatter corrections is useful and reliable for SPECT volume quantitation, and is a simple method for the in vivo measurement of organ volumes and radiation dosimetry in humans without the need for assumptions on organ concentration or size. Our data demonstrate the accuracy of this method when measuring a range of volumes that covers most situations encountered in a clinical setting. We believe that this method would replace the actual method used in clinical routine based on planar imaging [25]. It is difficult to verify this method in vivo, as there is no similar approach to estimation of thyroid volume in situ in humans. This study was limited to some clinical cases and a phantom study with homogeneous activity distribution. Further 
evaluation of the effects of background and heterogeneity of radionuclide concentration on the accuracy of this method and application of more sophisticated attenuation and scatter correction methods are still required.

\section{ACKNOWLEDGMENT}

The author would like to gratefully acknowledge the staff of the Departments of Radiation Physics of Malmö General Hospital and Lund University for their technical support and discussions during the conduct of the research. The author also thanks the reviewers for helping to improve the clarity of presentation.

\section{REFERENCES}

[1] G. Iosilevsky, O. Israel, A. Frenkel, E. Even-Sapir, S. Benhaim, S. Front, G. M. Kolodny, and D. Front, "A practical SPECT technique for quantitation of drug delivery to human tumors and organ absorbed radiation dose," Sem. Nucl. Med., vol. 19, pp. 33-46, 1989.

[2] S-E. Strand and M. Ljungberg, "Absorbed dose planning in radionuclide therapy based on Quantitative SPECT," Antibody Immunoconj. Radiopharm., vol. 4, pp. 673-680, 1991.

[3] M. A. Flower, T. Schlesinger, P. J. Hinton, I. Adam, A. M. Masoomi, M. A. Elbelli, R. J. Ott, V. R. McCready, and C. L. Harmer, "Radiation dose assessment in radioiodine therapy 2-Practical implementation using quantitative scanning and PET, with initial results on thyroid carcinoma," Radiother. Oncol., vol. 15, pp. 345-357, 1989.

[4] M. K. Kan and B. G. Hopkins, "Measurement of liver volume by emission computed tomography," J. Nucl. Med., vol. 20, pp. 514-520, 1979.

[5] B. Shapiro, L. Rigly, and K. E. Britton, "The assessment of thyroid volume with single photon emission tomography," Nucl. Med. Commun., vol. 1 , pp. 33-36, 1980.

[6] W. N. Tauxe, F. Soussaline, and A. E. Todd-Pokropek, "Determination of organ volume by single photon emission tomography," J. Nucl. Med., vol. 23, pp. 984-987, 1982.

[7] J. Kawamura, H. Itoh, O. Yoshida, T. Fujita, and K. Torizukak, "In vivo estimation of renal volume using a rotating gamma camera for ${ }^{99 m} \mathrm{Tc}$ dimercaptosuccinic acid renal imaging," Eur. J. Nucl. Med., vol. 9, pp. 168-172, 1984.

[8] L. T. Kircos, J. E. Carey, and J. W. Keyes, "Quantitative organ visualization using SPECT," J. Nucl. Med., vol. 28, pp. 334-341, 1987.

[9] M. A. King, D. T. Long, and A. B. Brill, "SPECT volume quantitation Influence of spatial resolution, source size and shape, and voxel size," Med. Phys., vol. 18, pp. 1016-1024, 1991.
[10] D. T. Long, M. A. King, and J. Sheehan, "Comparative evaluation of image segmentation methods for volume quantitation in SPECT," Med. Phys., vol. 19, pp. $483-489,1992$.

[11] N. Otsu, "A threshold selection method from gray-level histograms," IEEE Trans. Syst., Man. Cybern., vol. 9, pp. 62-66, 1979.

[12] NEMA, Standards for Performance Measurements of Scintillation Cameras, National Electrical Manufacturers Association, Washington D.C 1986.

[13] J. Mas, R. B. Younes, and R. Bidet "Improvement of quantification in SPECT studies by scatter and attenuation compensation," Eur. J. Nucl. Med., vol. 15, pp. 351-356, 1989.

[14] L. T. Chang, "A method for attenuation correction in radionuclide computed tomography," IEEE Trans. Nucl. Sci., vol. 25, pp. 638-643, 1978.

[15] M. Ljungberg and S-E. Strand, "A Monte Carlo program for the simulation of scintillation camera characteristics," Comp. Meth. Prog. Biomed., vol. 29, pp. 257-272, 1989.

[16] R. J. Jaszczak, K. L. Greer, C. E. Floyd, C. C. Harris, and R. E. Coleman, "Improved SPECT quantification using compensation for scattered photons," J. Nucl. Med., vol. 25, pp. 893-900, 1984.

[17] L. Mortelmans, J. Nuyts, G. Van Pamel, V. Van den Maegdenbergh, M. De Roo, and P. Suetens, "A new thresholding method for volume determination by SPECT," Eur. J. Nucl. Med., vol. 12, pp. 284 290, 1986.

[18] P. H. Pretorius, A. Van Aswegen, C. P. Herbst, and M. Lötter, "The effects of different correction techniques on absolute volume determination with SPECT using a threshold edge detection method," Med. Phys. vol. 18, pp. 390-393, 1991

[19] K. Murase, S. Tanada, Y. Yasuhara, H. Mogami, A. lio, and K. Hamamoto, "SPECT volume measurement using an automatic threshold selection method combined with a V filter," Eur. J. Nucl. Med., vol. 54, pp. $21-25,1989$.

[20] S. Webb, M. A. Flower, R. J. Ott, M. D. Broderick, A. P. Long B. Sutton, and V. R. McCready, "Single photon emission computed tomographic imaging and volume estimation of the thyroid using fanbeam geometry," Br. J. Radiol., vol. 59, pp. 951-955, 1986.

[21] J. Palmer and P. Wollmer, "Pinhole emission computed tomography: Method and experimental evaluation," Phys. Med. Biol., vol. 3, pp. $339-350,1990$

[22] P. K. Leichner, N-C. Yang, B. W. Wessels, W. G. Hawkins, S. E. Order, and $\mathbf{J}$. L. Klein, "Dosimetry and treatment planning in radioimmunotherapy," in Frontiers of Radiation Therapy and Oncology, J. M. Vaeth and J. M. Meyer, Eds. Basel: Karger Verlag, 1990, pp. 109-120.

[23] O. Basset, G. Gimenez, J. L. Mestas, D. Gathignol, and M. Devonec, "Volume measurement by ultrasonic transverse or sagittal cross-sectional scanning," Ultrasound in Med. \& Biol., vol. 17, pp. 291-296, 1991.

[24] S. Glickman, "Determination of organ volume with SPECT," Siemens Dialogos, vol. 3, pp. 24-37, 1987.

[25] H. Zaidi, "Comparative methods for quantifying thyroid volume using planar imaging and SPECT," J. Nucl. Med., to be published. 